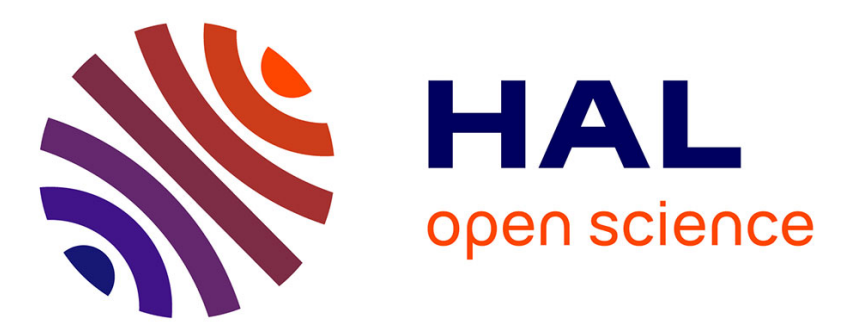

\title{
Effet des préférences individuelles sur la réussite à long terme des incitations financières à la réalisation d'objectifs de santé
}

David Crainich

\section{- To cite this version:}

David Crainich. Effet des préférences individuelles sur la réussite à long terme des incitations financières à la réalisation d'objectifs de santé. Revue d'Economie Politique, 2019, 129 (4), pp.447-465. 10.3917/redp.294.0447 . hal-02108283

\section{HAL Id: hal-02108283 \\ https://hal.science/hal-02108283}

Submitted on 18 Oct 2021

HAL is a multi-disciplinary open access archive for the deposit and dissemination of scientific research documents, whether they are published or not. The documents may come from teaching and research institutions in France or abroad, or from public or private research centers.
L'archive ouverte pluridisciplinaire HAL, est destinée au dépôt et à la diffusion de documents scientifiques de niveau recherche, publiés ou non, émanant des établissements d'enseignement et de recherche français ou étrangers, des laboratoires publics ou privés. 


\title{
Effet des préférences individuelles sur la réussite à long terme des incitations financières à la réalisation d'objectifs de santé
}

\author{
David Crainich \\ CNRS (LEM UMR 9221) et IÉSEG School of Management
}

\section{Introduction}

Les politiques publiques mises en œuvre ces dernières années afin d'infléchir les comportements néfastes à la santé ne semblent pas atteindre leurs objectifs. Dans son dernier rapport sur la santé en Europe, l'OMS estime ainsi que le tabagisme, la consommation d'alcool, le surpoids et l'obésité atteignent des niveaux alarmants: $30 \%$ de la population fume, $59 \%$ de la population est obèse ou en surpoids et les Européens consomment 11 litres d'alcool par personne et par an (Organisation Mondiale de la Santé, 2017). Les effets de ces comportements sur la santé sont importants : le tabagisme augmente la probabilité de développer des affections pulmonaires, l'obésité accroît le risque de diabète et les deux comportements sont des facteurs de risque majeurs de nombreux cancers et des maladies cardiovasculaires (Organisation Mondiale de la Santé, 2017).

Les politiques visant à limiter les comportements néfastes à la santé se heurtent au décalage qui existe entre des efforts qui doivent être effectués immédiatement et des bénéfices qui sont incertains, intangibles et différés. Des incitations financières accordées lorsque certains objectifs de santé (cessation du tabagisme, perte de poids,...) sont atteints permettent d'associer un avantage sûr, quantifiable et immédiat à des comportements sains. De nombreux travaux empiriques ont montré que de telles incitations étaient à même de provoquer ou de renforcer des modifications de comportements. L'efficacité de ces incitations financières a été mise en évidence dans la littérature, que ce soit pour stimuler l'arrêt du tabagisme (voir Halpern et al. (2015) pour une étude sur la population générale ou Tappin et al. (2015) pour une expérience spécifiquement menées avec des femmes enceintes), pour encourager la perte de poids (voir par exemple Volpp et al. (2008) ou John et al. (2011)) ou pour promouvoir l'activité physique (Finkelstein et al. (2008)). Cette efficacité a toutefois aussi été remise en cause lorsqu'elle est considérée après la fin du programme (voir PaulEbhohimhen et Avenell (2008) ou Spahn et al. (2011)). L'objectif de notre travail est de proposer une première approche théorique de cette question en vue de mettre en évidence les conditions sous lesquelles la réussite des programmes d'incitations financières aux objectifs de santé est favorisée. Nous nous focalisons plus particulièrement sur la distinction qui existe entre la propension à adopter des comportements sains à court terme (c'est-à-dire pendant la durée du programme) et à long terme (au-delà du programme).

Afin de déterminer les éléments favorisant la réussite de ces incitations financières, nous basons notre modèle sur les travaux en théorie du risque qui ont examiné les comportements de prévention. Depuis la contribution originale d'Ehrlich et Becker (1972) et les travaux qui s'en sont inspirés pour modéliser les risques de santé (voir par exemple Menegatti (2014) ou Courbage et Rey (2015) pour ne citer que les plus récents), la littérature en théorie du risque considère que les activités de 
prévention ont des effets directs et déterminés sur la probabilité de maladie (lorsqu'il s'agit de la prévention primaire) ou sur sa gravité (si la prévention secondaire est analysée). Si cette hypothèse peut être considérée comme plausible pour des risques financiers ou pour des risques d'accidents de la circulation, de cambriolages ou d'incendie, le lien qui existe entre l'effort et le bénéfice de la prévention peut être différent lorsque des risques de santé sont en jeu. En effet, la réduction de la probabilité de maladie peut aussi transiter par la modification de certains comportements (consommation de tabac et d'alcool, obésité, sédentarité,...) dont le succès ne dépend pas uniquement de la volonté de l'individu mais plus généralement du contexte et de l'environnement socio-économique dans lequel il évolue. L'originalité de notre travail est de prendre en compte cette dimension: au lieu de supposer qu'il suffit de fournir un effort individuel de prévention afin de réduire la probabilité de maladie, nous considérons que cet effort permet éventuellement de modifier un comportement qui, à son tour, réduit la probabilité d'une maladie qui pourrait se déclarer dans le futur. En cela, nous nous rapprochons des modèles adoptés par Jindapon et Neilson (2007) et par Crainich, Eeckhoudt et Menegatti (2016) dans lesquels l'effort ne conduit pas directement à un résultat favorable ou défavorable mais augmente la probabilité de faire face à une distribution plus favorable au sens de la dominance stochastique d'ordre $n$. Notre modélisation part du même principe mais est adaptée afin d'analyser l'efficacité des programmes d'incitations financières à la limitation des comportements néfastes à la santé. D'une part, alors que l'effort est unique dans les modèles de Jindapon et Neilson (2007) et de Crainich, Eeckhoudt et Menegatti (2016), nous scindons l'effort total en un effort de court terme réalisé uniquement durant la période du programme d'incitation et un effort de long terme réalisé après la fin du programme. Le premier débouche sur un paiement et réduit la pénibilité des efforts entrepris ultérieurement (les efforts de long terme) si l'objectif comportemental défini dans le programme est atteint, alors que le second augmente la probabilité d'adopter durablement un comportement favorable à la santé. D'autre part, alors que Jindapon et Neilson (2007) et Crainich, Eeckhoudt et Menegatti (2016) considèrent uniquement des risques financiers, nous considérons que l'utilité des individus dépend de deux arguments: la richesse et la qualité de vie. Cette spécification permet d'intégrer le fait que les comportements sains sont stimulés à court terme par une incitation financière mais requièrent des efforts non-monétaires et - lorsqu'ils sont poursuivis de façon durable - réduisent la probabilité de maladie.

Dans ce contexte nous montrons que lorsque la richesse et la qualité de vie sont complémentaires (c'est-à-dire si l'amélioration de la qualité de vie augmente l'utilité marginale de la richesse; la dérivée croisée de la fonction d'utilité est dans ce cas positive), les programmes d'incitations financière peuvent susciter des comportements opposés à ceux pour lesquels ils ont été introduits. A l'inverse, notre travail montre que ces programmes favorisent l'adoption de comportements sains pendant et après le programme lorsque l'amélioration de la qualité de vie réduit ou laisse inchangée l'utilité marginale de la richesse (la dérivée croisée de la fonction d'utilité est négative ou nulle).

L'article est organisé comme suit. Les hypothèses du modèle et les notations sont établies à la section 2. Nous décrivons à la section 3 une situation particulière où les programmes d'incitations financières conduisent les individus à faire moins d'efforts à long terme afin de réduire leurs risques de santé. Afin de comprendre les mécanismes qui engendrent de tels effets, nous modélisons à la 
section 4 les niveaux d'efforts mis en oeuvre pendant et après la durée du programme. Cela nous permet d'analyser les effets des incitations financières octroyées lorsqu'un objectif de comportement est atteint (section 5) et de mettre ensuite en évidence la façon dont l'interaction entre la richesse et la qualité de vie affecte l'efficacité de ces incitations (section 6). Nous concluons I'article à la section 7.

\section{Hypothèses et notations}

Nous considérons la situation d'un individu qui maximise son espérance d'utilité sur trois périodes ( $t=0,1$ et 2 ). Son utilité dépend de deux arguments : sa richesse $(w)$ et un indice de sa qualité de vie $(h)$. Cette dernière variable englobe l'ensemble des éléments non-financiers ayant un effet sur son bien-être : son état de santé ${ }^{1}$, son environnement, les efforts qui affectent sa qualité de vie,... Sa fonctions d'utilité aux trois périodes s'écrit $u(w, h)$ et nous émettons les hypothèses habituelles à propos de cette dernière : I'utilité est croissante avec la richesse $\left(u_{1}>0\right)$ et avec la qualité de vie $\left(u_{2}>0\right)$; l'individu a de l'aversion vis-à-vis des risques de richesse $\left(u_{11}<0\right)$ et des risques de qualité de vie $\left(u_{22}<0\right)^{2}$. La richesse initiale et la qualité de vie initiale de l'individu sont respectivement dénotées $w$ et $h$ (elles sont invariables d'une période à l'autre). Le taux de préférence pour le présent est supposé nul ${ }^{3}$.

A la période $t=0$, l'individu participe à un programme de santé qui octroie un paiement (dénoté $s$ ) lorsqu'un objectif comportemental (arrêt du tabagisme, perte de poids, pratique régulière d'exercice physique,...) est atteint à l'issue d'une période prédéterminée ${ }^{4}$. Atteindre l'objectif nécessite des efforts (dénotés $e$ ) qui réduisent la qualité de vie de l'individu ${ }^{5}$. Ces efforts ne lui garantissent pas d'atteindre son objectif mais lui permettent d'en augmenter la probabilité de succès. La probabilité de ne pas atteindre l'objectif est dénotée $p(e)$ et, dans la mesure où seules deux situations sont envisagées (succès ou échec), la probabilité de l'atteindre est dénotée $1-p(e)$. Par conséquent, $p^{\prime}(e)<0$ (les efforts réduisent la probabilité d'échec) et nous supposons que $p^{\prime \prime}(e) \geq 0$ (l'effet marginal de chaque effort supplémentaire sur la probabilité d'atteindre l'objectif est décroissant). $\mathrm{Si}$ l'objectif de comportement est atteint, deux types de bénéfices sont associés aux efforts effectués à la période 0 : d'une part, l'individu reçoit le paiement $s$; d'autre part, les efforts effectués ultérieurement au programme (en $t=1$ ) lui seront moins pénibles. Plus précisément, lorsque l'objectif a été atteint à la période 0 , les efforts effectués à la période 1 sont dénotés $z_{S}$ et le coût exprimé en terme de qualité de vie - qui y est associé est représenté par la fonction $\alpha(e, z)$ à propos

\footnotetext{
${ }^{1}$ Les résultats que nous mettons en évidence dans l'article ne sont pas modifiés si nous considérons que l'état de santé n'est pas englobé dans l'indice de qualité de vie mais constitue un argument à part entière de la fonction d'utilité.

${ }^{2}$ La notation suivante est adoptée: $u_{1}$ et $u_{11}$ désignent respectivement les dérivées premières et secondes de l'utilité par rapport à la richesse; $u_{2}$ et $u_{22}$ désignent respectivement les dérivées premières et secondes de l'utilité par rapport à la qualité de vie; $u_{12}$ désigne la dérivée croisée de l'utilité par rapport à la richesse et à la qualité de vie.

${ }^{3}$ Le taux de préférence pour le présent joue un rôle crucial lorsque des décisions qui ont des conséquences à long terme sont analysées. Dans notre modèle, il ne modifie cependant pas la façon dont l'interaction entre la richesse et la qualité de vie ( $c$ 'est-à-dire le signe de $u_{12}$ ) influence l'efficacité à long terme des programmes d'incitations financières aux objectifs de comportement.

${ }^{4}$ Nous ne considérons pas ici les situations où les récompenses financières sont octroyées graduellement.

${ }^{5}$ Dans le modèle, les efforts n'ont pas de conséquences financières. Cette hypothèse est discutée et modifiée dans l'annexe 1 où nous supposons que l'effort permet à l'individu d'économiser de l'argent (parce que par exemple, l'individu n'achète plus de tabac lorsqu'il a arrêté de fumer).
} 
de laquelle les hypothèses suivantes sont effectuées ${ }^{6}: \alpha_{e}(e, z)<0 ; \alpha_{z}(e, z)>0 ; \alpha_{z z}(e, z)>$ $0 ; \alpha_{e z}(e, z)<0$. Cette dernière hypothèse exprime le fait que lorsque l'objectif de comportement est atteint, la pénibilité de chaque effort décroit avec l'intensité des efforts préalablement effectués (les premiers efforts entrepris afin de modifier les comportements liés à la santé étant les plus pénibles). Lorsque l'objectif du programme n'a pas été atteint, $\varphi\left(z_{s}\right)$ représente la pénibilité des efforts effectués à la période 1 (dénotés $\left.z_{\varsigma}\right)^{7}$ : la perte de qualité de vie à la période 1 ne dépend donc que de $z_{s}$ et pas des efforts entrepris précédemment ${ }^{8}$.

Les efforts effectués à la période 1 ne garantissent pas l'adoption d'un comportement sain à long

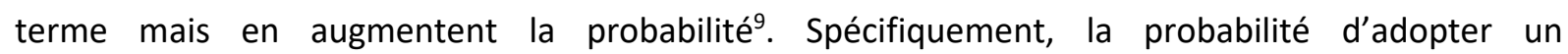
comportement néfaste et celle d'adopter un comportement sain à la fin de la période 1 sont respectivement dénotées $q(z)$ et $1-q(z)$ (avec $z=z_{s}$ ou $z=z_{s}$ en fonction de la réalisation de l'objectif à la période 0).

Au début de la période 2, si l'individu a atteint son objectif de santé à long terme (il est devenu durablement non-fumeur, il maintient son poids idéal dans la durée,...), la distribution de sa qualité de vie $(\tilde{L})$ est plus favorable que si son objectif de long terme $n^{\prime}$ est pas atteint (cette distribution est alors dénotée $\widetilde{H}) . E U(\widetilde{L})$ et $E U(\widetilde{H})$ sont les espérances d'utilité associées à ces deux distributions. A titre d'exemple, si l'on suppose que devenir durablement non-fumeur réduit la probabilité (de $p_{H}$ à $p_{L}$ avec $p_{H}>p_{L}$ ) de développer une maladie dont la sévérité est dénotée $M$, les distributions de qualité de vie auxquelles l'individu fait face à la période 2 s'écrivent $\tilde{L}=\left(h, h-M ; 1-p_{L}, p_{L}\right)$ s'il a atteint son objectif à long terme et $\widetilde{H}=\left(h, h-M ; 1-p_{H}, p_{H}\right)$ dans le cas contraire. Notre analyse est toutefois plus générale dans la mesure où elle s'étend aux situations où atteindre l'objectif de comportement à la fin de la période 1 affecte la sévérité et non pas la probabilité de maladie ou aux situations où atteindre cet objectif affecte à la fois les deux dimensions du risque de maladie (probabilité et sévérité).

\footnotetext{
${ }^{6}$ Nous utilisons la notation suivante: si l'objectif comportemental a été atteint, $\alpha_{e}$ et $\alpha_{z}$ désignent les dérivées de la réduction de la qualité de vie par rapport aux efforts effectués respectivement aux périodes 0 et 1 et $\alpha_{e z}$ désigne la dérivée croisée de la réduction de la qualité de vie par rapport à ces deux mêmes efforts.

${ }^{7}$ Si l'objectif comportemental n'a pas été atteint, $\varphi^{\prime}$ et $\varphi^{\prime \prime}$ désignent respectivement les dérivées premières et secondes du coût de l'effort et nous supposons que $\varphi^{\prime}>0$ et $\varphi^{\prime \prime}>0$.

${ }^{8}$ Par exemple, la pénibilité du renoncement à fumer à la période 1 n'est réduite par l'effort effectué à la période 0 que si ce dernier a effectivement conduit à l'arrêt du tabagisme. Cette hypothèse est sujette à discussion : on pourrait supposer que le coût psychologique de l'effort décroît lorsque les individus ont déjà essayé d'arrêter ou, à l'inverse, que le découragement engendré par un échec rend les tentatives ultérieures plus pénibles. Dans ces deux cas de figure, le modèle conduit à des résultats ambigus dans la mesure où les trois variables endogènes interagissent et qu'il est alors difficile d'exploiter le théorème des fonctions implicites. Afin de rendre le modèle plus manipulable, nous supposons qu'en cas $\mathrm{d}^{\prime}$ 'échec lors de la tentative effectuée à la période 0 , l'individu se retrouve dans sa situation de départ (ses efforts antérieurs sont sans effets).

${ }^{9}$ Seuls les efforts effectués durant la période 1 - supposée plus longue que la période 0 qui ne dure que le temps du programme - accroissent directement la probabilité d'adopter durablement des comportements bénéfiques à la santé. Cette hypothèse facilite la résolution du modèle dans la mesure où elle permet d'écrire la probabilité d'adopter durablement un comportement sain uniquement en fonction de l'effort de long terme (et non pas de l'effort effectué pendant la durée du programme). En cela, notre hypothèse cadre avec l'objectif de ces programmes qui sont mis en place afin de déclencher des comportements durablement sains mais sont sans effets si les individus reprennent leurs anciennes habitudes dès qu'ils ont empoché la récompense financière.
} 
La figure 1 représente la situation d'un individu participant au programme :

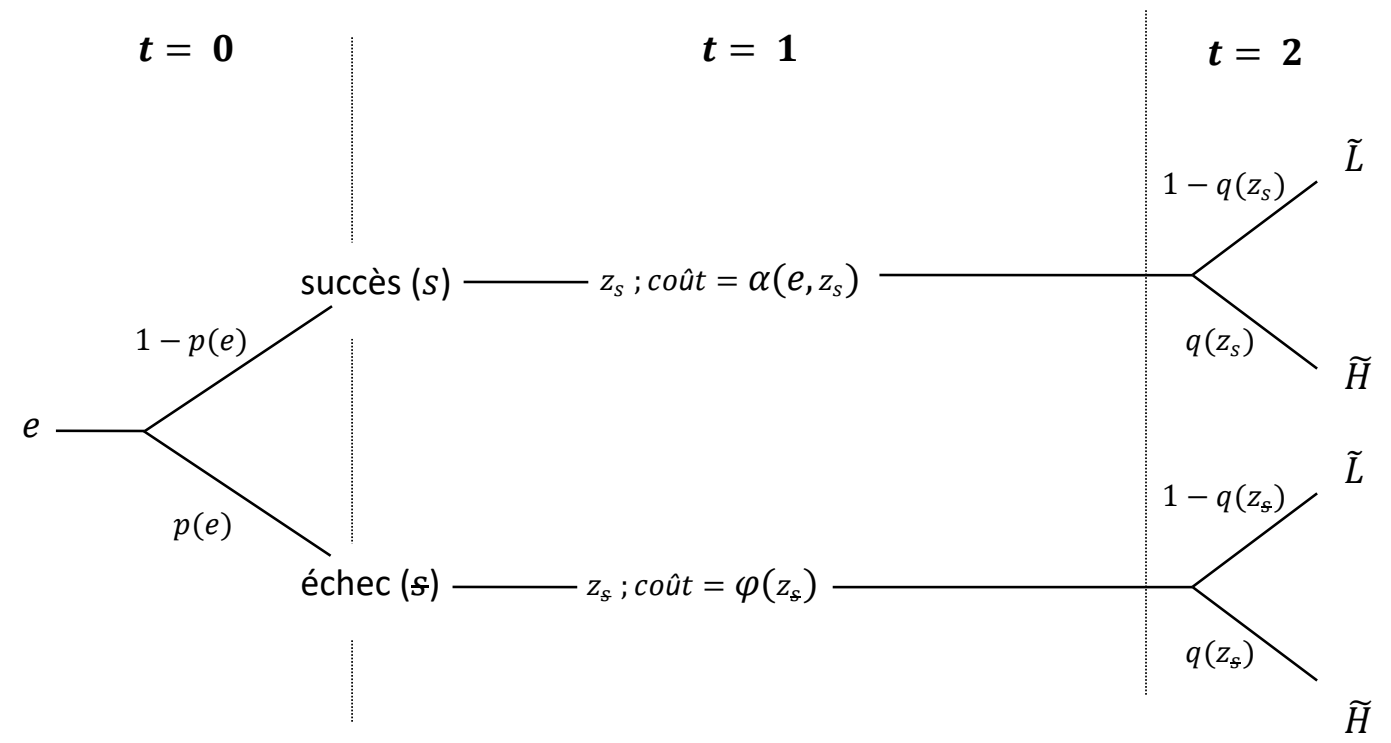

Figure 1

\section{Un cas particulier}

Nous exposons dans cette section un cas où les hypothèses effectuées à la section 2 sont telles que les programmes d'incitations financières à la réalisation d'objectifs comportementaux ont des effets à long terme opposés à ceux pour lesquels ils ont été introduits.

Nous supposons que les fonctions d'utilité sont invariables d'une période à l'autre et s'écrivent:

$$
u(w, h)=(w h)^{0,95}
$$

Cette fonction d'utilité possède les caractéristiques désirées, à savoir : $u_{1}=0,95 w^{-0,05} h^{0,95}>0$; $u_{11}=-0.0475 w^{-1,05} h^{0,95}<0 ; u_{2}=0,95 w^{0,95} h^{-0,05}>0 ; u_{22}=-0,0475 w^{0,95} h^{-1,05}<0$;

Dans la mesure où $u_{12}=0,9025 w^{0,95} h^{0,95}>0$, cette fonction représente des préférences telles que la richesse et la qualité de vie sont des biens complémentaires.

Les niveaux initiaux de richesse $(w)$ et de qualité de vie $(h)$ et la perte de qualité de vie en cas de maladie à la période $2(M)$ prennent les valeurs suivantes : $w=50 ; h=50 ; M=30$.

Les probabilités de ne pas atteindre son objectif comportemental à la fin de la période 0 et à la fin de la période 1 sont respectivement définies par : $p(e)=1-\frac{\sqrt{e}}{10}$ et $q(z)=1-\frac{\sqrt{z}}{10}$.

Le coût de l'effort de prévention à la période 1 lorsque l'objectif de comportement a été atteint à la période 0 est défini par $\alpha(e, z)=\frac{z^{2}}{20}-\frac{e z}{50}$, de sorte que $\alpha_{e}=-\frac{z}{50}<0 ; \alpha_{z}=\frac{z}{10}-\frac{e}{50}<0$ (pour autant que $z>\frac{e}{5}$; cette condition est respectée dans notre illustration); $\alpha_{e z}=-\frac{1}{50}<0$. Ce même 
coût de l'effort à la période 1 lorsque l'objectif de comportement n'a pas été atteint à la période 0 est défini par: $\varphi\left(z_{s}\right)=\frac{z_{s}^{2}}{5}$, de sorte que $\varphi^{\prime}=\frac{2 z_{s}}{5}>0$ et $\varphi^{\prime \prime}=\frac{2}{5}>0$.

Enfin, les probabilités de développer la maladie lorsque l'objectif comportemental de long terme est atteint et lorsqu'il ne l'est pas prennent respectivement les valeurs suivantes : $p_{L}=0,3$ et $p_{H}=0,7$.

Compte tenu de ces hypothèses, le tableau ci-dessous indique les niveaux d'efforts effectués aux périodes 0 et 1 compte tenu de 4 niveaux de paiement lorsque l'objectif du programme est atteint.

\begin{tabular}{|ccccc|}
\hline $\mathbf{s}$ & $\mathbf{0}$ & $\mathbf{5}$ & $\mathbf{1 0}$ & $\mathbf{1 5}$ \\
\hline $\mathbf{e}$ & 0,002 & 0,08 & 0,29 & 0,63 \\
$\mathbf{z}_{\mathbf{s}}$ & 4,03 & 3,91 & 3,83 & 3,77 \\
$\mathbf{z}_{\mathbf{s}}$ & 1,6 & 1,6 & 1,6 & 1,6 \\
\hline
\end{tabular}

Sans incitation financière, l'individu effectue un effort réduit à la période 0 . L'introduction et les différentes augmentations du paiement l'amènent à accroître son effort. Simultanément, l'effort à la période 1 diminue avec le montant de l'incitation. Nous nous retrouvons donc ici en présence d'une incitation financière qui stimule l'effort pendant la période du programme mais qui le réduit au-delà du programme. Dans la mesure où ce n'est pas l'effort effectué durant la courte période que dure le programme mais bien celui consenti à plus long terme qui a un impact sur la probabilité de maladie, l'exemple décrit une situation où le programme n'atteint pas son objectif.

Nous décrivons aux sections 4,5 et 6 les mécanismes qui conduisent à cet effet non désiré et les conditions sous lesquelles il peut se produire.

\section{Les efforts de comportements à l'équilibre}

Compte tenu de hypothèses établies à la section 2, l'individu maximise l'utilité intertemporelle $A$ en choisissant simultanément les niveaux d'efforts $e, z_{S}$ et $z_{S}$ :

$$
\begin{gathered}
A=u(w, h-e)+(1-p(e))\left[u\left(w+s, h-\alpha\left(e, z_{s}\right)\right)+\left(1-q\left(z_{s}\right)\right) E U(\widetilde{L})+q\left(z_{s}\right) E U(\widetilde{H})\right] \\
+p(e)\left[u\left(w, h-\varphi\left(z_{s}\right)\right)+\left(1-q\left(z_{s}\right)\right) E U(\widetilde{L})+q\left(z_{s}\right) E U(\widetilde{H})\right]
\end{gathered}
$$

L'utilité de l'individu durant la durée du programme (premier terme de $A$ ) dépend négativement de l'effort $e$ effectué afin de modifier son comportement en matière de santé. Le second terme de $A$ correspond à la situation de l'individus aux périodes 1 et 2 lorsqu'il a atteint son objectif comportemental à la fin du programme (ce qui se produit avec une probabilité $1-p(e)$ ). Dans ce cas, l'individu reçoit au début de la période 1 le montant $s$ et poursuit durant cette période des efforts $z_{s}$ dont la pénibilité s'écrit $\alpha\left(e, z_{s}\right)$. Ces efforts lui permettent d'atteindre son objectif comportemental à long terme avec une probabilité $1-q\left(z_{s}\right)$ et son risque de maladie est dans ce cas relativement bas à la période $2(E U(\tilde{L}))$. Son objectif de long terme n'est pas atteint avec une probabilité $q\left(z_{S}\right)$ et son risque de maladie est alors relativement élevé $(E U(\widetilde{H}))$ à la période 2 . Le dernier terme de $A$ correspond à la situation de l'individus aux périodes 1 et 2 lorsqu'il n'a pas atteint son objectif comportemental (la probabilité de cet évènement est $p(e)$ ) et s'interprète de la même façon. A la période 1, l'individu poursuit ses efforts (dont la pénibilité $q\left(z_{s}\right)$ ne dépend cette fois pas des efforts effectués préalablement) afin d'atteindre son objectif de comportement à long terme. Cet 
objectif est atteint avec une probabilité $1-q\left(z_{s}\right)$ et son risque de maladie à la période 2 est alors relativement faible $(E U(\tilde{L}))$. S'il n'atteint pas son objectif à long terme, ce qui se produit avec une probabilité $q\left(z_{s}\right)$ ), son risque de maladie à la période 2 est relativement élevé $(E U(\widetilde{H})$ ).

Afin de réduire la longueur des expressions, la notation suivante est adoptée :

$S=w+s, h-\alpha ; E U(S)=u(S)+\left(1-q\left(z_{S}\right)\right) E U(\tilde{L})+q\left(z_{S}\right) E U(\widetilde{H}) ;$

$\S=w, h-\varphi ; E U(\S)=u(\S)+\left(1-q\left(z_{s}\right)\right) E U(\tilde{L})+q\left(z_{s}\right) E U(\widetilde{H})$

Les conditions de premier ordre qui définissent les niveaux optimaux d'efforts $e^{*}$, $\mathrm{z}_{S}^{*}$ et $z_{S}^{*}$ aux périodes 0 et 1 , respectivement dénotées $A_{e}\left(=\frac{\delta A}{\delta e}\right), A_{z_{S}}\left(=\frac{\delta A}{\delta z_{s}}\right)$ et $A_{z_{s}}\left(=\frac{\delta A}{\delta z_{s}}\right)$ s'écrivent :

$A_{e}=-u_{2}\left(w, h-e^{*}\right)-p^{\prime}\left(e^{*}\right)[E U(S)-E U(S)]-\left(1-p\left(e^{*}\right)\right) \alpha_{e}\left(e^{*}, z_{S}\right) u_{2}(S)=0$

$A_{z_{S}}=-\alpha_{z}\left(e, \mathrm{z}_{S}^{*}\right) u_{2}(S)-q^{\prime}\left(\mathrm{z}_{S}^{*}\right)[E U(\tilde{L})-E U(\widetilde{H})]=0$

$A_{z_{s}}=-\varphi^{\prime}\left(z_{s}^{*}\right) u_{2}(S)-q^{\prime}\left(z_{s}^{*}\right)[E U(\tilde{L})-E U(\widetilde{H})]=0$

Les conditions de second ordre de ce programme de maximisation sont supposées être respectées de sorte que les conditions de premier ordre ci-dessus définissent un maximum global ${ }^{10}$.

Les conditions de premier ordre mettent en évidence les mécanismes qui conduisent à limiter les comportements néfastes à la santé aux périodes 0 et 1 . Lorsque l'individu effectue un effort à la période 0 , son utilité diminue via la réduction de sa qualité de vie $\left(\alpha_{e}\left(e, z_{s}\right) u_{2}(S) ; 1^{\text {er }}\right.$ terme de $\left.A_{e}\right)$. En contrepartie, le bénéfice de cet effort est double: d'une part, il augmente de $-p^{\prime}(e)$ la probabilité d'obtenir le paiement $s$ qui lui permet de passer d'une espérance d'utilité $E U(S)$ à une espérance d'utilité $E U(S)$ à la période 1 ( $2^{\text {ème }}$ terme de $A_{e}$ ); d'autre part, cette augmentation de l'effort à la période 0 lui permet - si l'objectif est atteint à la fin de la période 0 , ce qui se produit avec une probabilité $1-p(e)$ - de réduire de $\alpha_{e}\left(e, z_{S}\right) u_{2}(S)$ la pénibilité des efforts effectués à la période 1 ( $3^{\text {ème }}$ terme de $A_{e}$ ).

Si l'individu a atteint son objectif à la fin de la période 0 et s'il effectue des efforts afin de modifier son comportement à la période 1 , son utilité diminue à cette période via la réduction de sa qualité de vie $\left(-\alpha_{z}\left(e, z_{s}\right) u_{2}(S) ; 1^{\text {er }}\right.$ terme de $\left.A_{z_{s}}\right)$. Ces efforts lui permettent d'augmenter de $-q^{\prime}\left(z_{s}\right)$ la probabilité de faire face à un risque de maladie faible à la période 2 et ainsi de passer d'une espérance d'utilité $E U(\widetilde{H})$ à une espérance d'utilité $E U(\widetilde{L})$ ( $2^{\text {ème }}$ terme de $A_{z_{S}}$ ).

S'il n'a pas atteint son objectif de comportement à la période 0 , l'effort de l'individu à la période 1 répond à la même logique. Toutefois, dans la mesure où la condition de premier ordre $A_{z_{s}}$ ne dépend ni du paiement $s$, ni des efforts $e$ et $z_{S}$, la valeur de $z_{s}^{*}$ n'est pas affectée par $s^{11}$. La caractérisation

\footnotetext{
${ }^{10}$ Lorsque des actions qui réduisent la probabilité d'un évènement sont examinées, les problèmes liés au respect des conditions de second ordre ont été mis en évidence par Ehrlich et Becker (1972). Depuis lors, il est fréquent dans cette littérature de supposer - soit explicitement (Jullien, Salanié et Salanié (1999); Eeckhoudt et Gollier (2005)), soit implicitement (Briys et Schlesinger (1990)) - que ces conditions sont respectées.

${ }^{11}$ Comme illustré par l'exemple proposé à la section 3 : la valeur de $z_{s}^{*}$ est de 1,6 indépendamment du niveau de $s$.
} 
des niveaux $e^{*}$ et $z_{s}^{*}$ à l'aide des équations $A_{e}$ et $A_{z_{s}}$ suffisent donc à déterminer les effets des programmes d'incitations financières sur les variables du modèle. Les sections 5 et 6 mettent en évidence les mécanismes qui favorisent le succès de ces programmes.

\section{Effet de l'incitation financière sur les efforts de comportements}

Le théorème des fonctions implicites à deux variables nous permet de déterminer formellement les effets simultanés des programmes d'incitations financières sur les efforts effectués aux périodes 0 et 1. Ces effets sont respectivement donnés par les deux expressions suivantes :

$$
\begin{aligned}
& \frac{d e}{d s}=\frac{1}{D}\left[-A_{z_{s} z_{s}} A_{e s}+A_{e z_{s}} A_{z_{S} s}\right] \\
& \frac{d z_{s}}{d s}=\frac{1}{D}\left[-A_{e e} A_{z_{S} s}+A_{e z_{s}} A_{e s}\right]
\end{aligned}
$$

où $A_{e e}$ et $A_{z_{s} z_{s}}$ sont les conditions de second ordre respectivement associée à $A_{e}$ et à $A_{z_{s}}$ et où $D=A_{e e} A_{z_{s} z_{s}}-\left(A_{e z_{s}}\right)^{2}$ est le déterminant de la matrice Hessienne. L'hypothèse que $e^{*}$ et $z_{s}^{*}$ définissent un maximum global (voir section 4) implique $A_{e e}<0, A_{z_{s} z_{s}}<0$ et $D>0$.

Afin de bien comprendre les mécanismes qui déterminent la réussite des programmes d'incitations financières, nous décomposons tous les effets de ces derniers sur les variables du modèle.

Nous déterminons dans un premier temps l'effet direct de l'incitation financière sur l'effort de comportement à la période $0, c^{\prime}$ est-à-dire sur la façon dont le paiement $s$ déplace la fonction de réaction $e\left(z_{S}\right)$. Cet effet est donné par l'expression :

$$
\frac{\delta^{2} A}{\delta e \delta s}=A_{e s}=-p^{\prime}\left(e^{*}\right) u_{1}(S)-\left(1-p\left(e^{*}\right)\right) \alpha_{e}\left(e^{*}, z_{S}\right) u_{12}(S)
$$

Compte tenu de la structure temporelle du modèle, le paiement modifie uniquement le bénéfice de l'effort $e$. Ce premier effet direct se decompose en deux sous-effets. Le premier sous-effet de $s$ sur $e$ est positif $\left(-p^{\prime}\left(e^{*}\right) u_{1}(S)\right)$ et incite donc à accroître l'effort: l'accroissement de $s$ permet effectivement de creuser l'écart de richesse entre les situations de succès et d'échec dans l'atteinte de l'objectif du programme. Le second sous-effet de $s$ sur $e$ dépend du signe de $u_{12}$. L'effort $e$ rend moins pénible l'effort $z_{S}$ effectué à la période ultérieure et ce bénéfice est proportionnel à l'utilité marginale de la qualité de vie (voir le $3^{\text {ème }}$ terme de $A_{e}$ ). Lorsque $s$ augmente, l'utilité marginale de la qualité de vie augmente si la richesse et la qualité de vie sont complémentaires $\left(u_{12}>0\right)$. Dans un tel cas, le second sous-effet de $s$ sur $e\left(-\left(1-p\left(e^{*}\right)\right) \alpha_{e}\left(e^{*}, z_{s}\right) u_{12}(S)\right)$ est aussi positif et les deux sous-effets vont dans le même sens ${ }^{12}$. Nous obtenons alors:

$$
u_{12} \geq 0 \Rightarrow A_{\text {es }}>0
$$

Lorsque $u_{12}<0$, les deux sous-effets de $s$ sur $e$ vont en directions opposées et le signe de $A_{e s}$ est indéterminé.

Le deuxième effet direct est celui de l'augmentation du paiement $s$ sur l'effort effectué à la période 1 (déplacement la fonction de réaction $z_{S}(e)$ ). Cet effet est donné par l'expression :

\footnotetext{
${ }^{12}$ Seul le premier sous-effet est à l'œuvre lorsque $u_{12}=0$ qui conduit donc directement à $A_{e s}>0$.
} 


$$
\frac{\delta^{2} A}{\delta z_{S} \delta s}=A_{z_{S} s}=-\alpha_{z}\left(e, \mathrm{z}_{S}^{*}\right) u_{12}(S)
$$

L'effet de l'augmentation de $s$ sur $z_{S}$ dépend uniquement de la façon dont la richesse modifie l'utilité marginale de la qualité de vie (signe de $u_{12}(S)$ ). La complémentarité entre la richesse et la qualité de vie $\left(u_{12}(S)>0\right)$ augmente l'utilité marginale de la qualité de vie à la période 1 lorsque l'incitation financière augmente. Dans un tel cas, l'effort $z_{S}$ est considéré par l'individu comme étant plus pénible et il en réduit donc l'intensité. L'inverse se produit lorsque $u_{12}<0$. Par conséquent, le sens du déplacement de la fonction de réaction $z_{S}(e)$ lorsque $s$ varie est uniquement déterminé par le signe de $u_{12}$ :

$$
u_{12} \gtreqless 0 \Leftrightarrow A_{z_{S} S} \lesseqgtr 0
$$

II nous reste à déterminer les effets que les variables $e$ et $z_{S}$ ont l'une sur l'autre (effets indirects). les pentes des fonctions de réaction $e\left(z_{S}\right)$ et $z_{S}(e)$ sont définies par l'expression $A_{e z_{S}}\left(=\frac{\delta^{2} A}{\delta e \delta z_{S}}\right)$.

$$
\begin{gathered}
A_{e z_{S}}=p^{\prime}(e) \alpha_{z}\left(e, z^{*}\right) u_{2}(S)+p^{\prime}(e) q^{\prime}\left(z_{S}\right)[E U(\widetilde{L})-E U(\widetilde{H})]-(1-p(e)) \alpha_{e z}\left(e, z^{*}\right) u_{2}(S) \\
+(1-p(e)) \alpha_{z}\left(e, z^{*}\right) \alpha_{e}\left(e, z^{*}\right) u_{22}(S)
\end{gathered}
$$

En utilisant la condition de premier ordre $A_{z_{s}}=0$, les deux premiers termes de $A_{e z_{s}}$ s'annulent, ce qui nous permet d'obtenir :

$$
A_{e z_{S}}=(1-p(e))\left[-\alpha_{e z}\left(e, z^{*}\right) u_{2}(S)+\alpha_{z}\left(e, z^{*}\right) \alpha_{e}\left(e, z^{*}\right) u_{22}(S)\right]>0
$$

Compte tenu des hypothèses formulées à la section 2 , les fonctions de réaction $e\left(z_{s}\right)$ et $z_{s}(e)$ sont donc toujours croissantes.

\section{L'effet de la richesse sur l'utilité marginale de la qualité de vie}

Les hypothèses effectuées jusqu'à présent ne nous permettent pas de déterminer les signes de $\frac{d e}{d s}$ et de $\frac{d z}{d s}$ de façon générale. Toutefois, nous pouvons mettre en évidence certains résultats en faisant des hypothèses sur le signe de $u_{12}$. Afin de montrer le role joué par l'interaction entre la richesse et la qualité de vie dans le succès des programmes d'incitations financières aux comportements bénéfiques à la santé, nous allons considérer séparément les trois situations potentielles, à savoir $u_{12}=0, u_{12}<0$ et $u_{12}>0$.

\subsection{Le richesse n'a pas d'effet sur l'utilité marginale de la qualité de vie $\left(u_{12}=0\right)$}

Lorsque $u_{12}=0$, le paiement $s$ n'a pas d'effet direct sur l'effort effectué à la période $1\left(E U_{z_{s} s}=0\right)$. Les effets des variations de l'incitation financière sur les efforts $e$ et $z_{S}$ sont alors donnés par:

$$
\begin{gathered}
\frac{d e}{d s}=\frac{-E U_{z_{s} z_{s}} E U_{e s}}{D}>0 \\
\frac{d z_{s}}{d s}=\frac{E U_{e z_{s}} E U_{e s}}{D}>0
\end{gathered}
$$

Lorsque $u_{12}=0$, l'augmentation du paiement $s$ conduit à un accroissement des efforts afin d'adopter un comportement sain à la fois pendant et après le programme. 


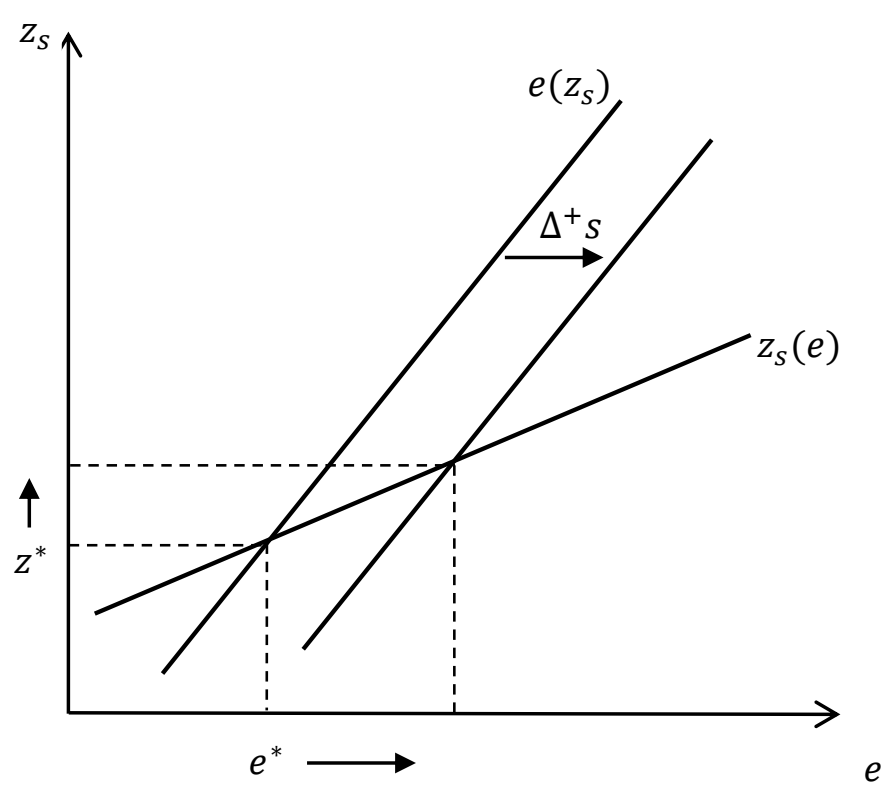

Figure 2

La situation est représentée à la figure 2. Seul l'effort effectué à la période 0 est directement affecté par l'introduction de l'incitation financière à l'objectif de santé (déplacement de $e\left(z_{s}\right)$ vers la droite). Les fonctions de réaction étant croissantes, le programme d'incitations financières a donc pour effet d'augmenter simultanément $e^{*}$ et $\mathrm{z}_{S}^{*}$.

\subsection{Le richesse réduit l'utilité marginale de la qualité de vie $\left(u_{12}<0\right)$}

La figure 3 décrit l'effet de l'introduction de l'incitation financière lorque $u_{12}(S)<0$.

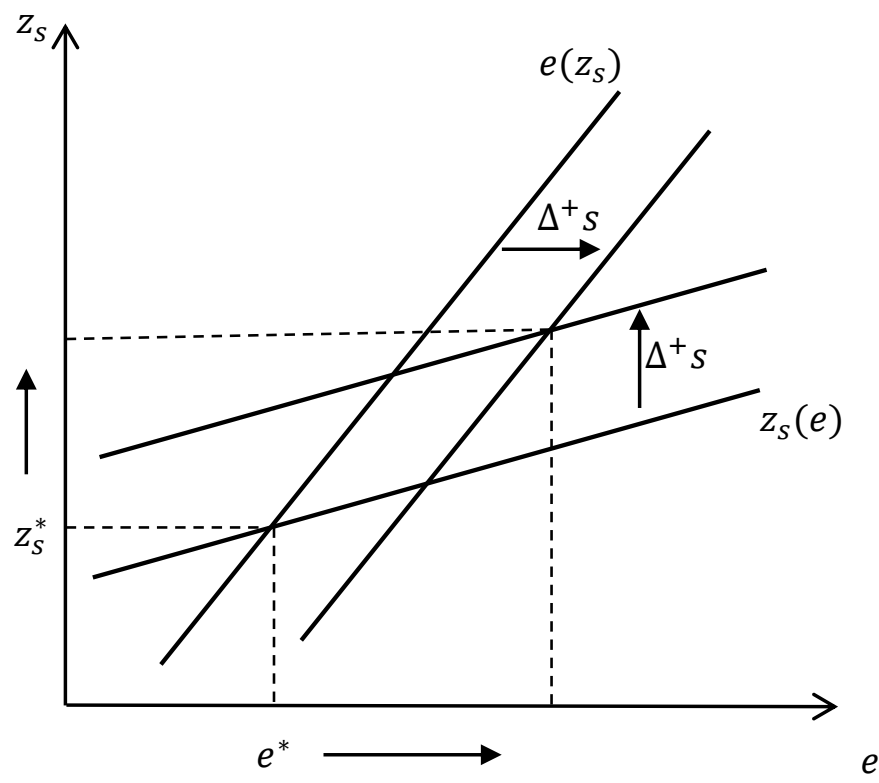

Figure 3 
Nous faisons l'hypothèse que la subvention $s$ accroît l'effort à la période 0 (déplacement de $e\left(z_{s}\right)$ vers la droite) : cela revient à supposer que le programme d'incitation financière atteint au moins son objectif à court terme, à savoir stimuler les comportements bénéfiques à la santé pendant la durée du programme. Puisque $u_{12}(S)<0$ implique que l'accroissement de $s$ déplace $z_{S}(e)$ vers la haut et que les fonctions de réactions sont - quel que soit le signe de $u_{12}$ - croissantes, tous les effets vont dans le même sens : l'effet direct de l'augmentation de l'incitation financière est positif sur l'effort effectué pendant et après le programme et, par ailleurs, les mouvements de ces deux variables se renforcent.

Si l'augmentation de $s$ a un effet positif sur $e$, le programme d'incitations financières sera donc efficace à la fois pendant et après sa mise en place si $u_{12}(S)<0$.

\subsection{Le richesse augmente l'utilité marginale de la qualité de vie $\left(u_{12}>0\right)$}

Enfin, nous montrons que $u_{12}(S)>0$ est l'hypothèse (nécessaire mais pas suffisante) cruciale qui conduit à des effets potentiellement contre-productifs à long terme des programmes d'incitations financières à la réalisation d'objectifs de santé. Comme indiqué à la section $5, u_{12}(S)>0$ implique que l'effet direct de l'accroissement de $s$ est positif sur $e$ et négatif sur $z_{s}$. En tenant compte des effets indirects toujours positifs entre les efforts effectués aux deux périodes, l'effet total de l'augmentation de $s$ sur $e$ et $z_{s}$ est dès lors ambigu. En effet :

$$
\begin{aligned}
& \frac{d e}{d s}=\frac{1}{D}\left[-E U_{z_{s} z_{s}} E U_{e s}+E U_{e z_{s}} E U_{z_{S} s}\right] \gtreqless 0 \\
& \frac{d z_{s}}{d s}=\frac{1}{D}\left[-E U_{e e} E U_{z_{s} s}+E U_{e z_{s}} E U_{e s}\right] \gtreqless 0
\end{aligned}
$$

La figure 4 décrit une de ces possibilités :

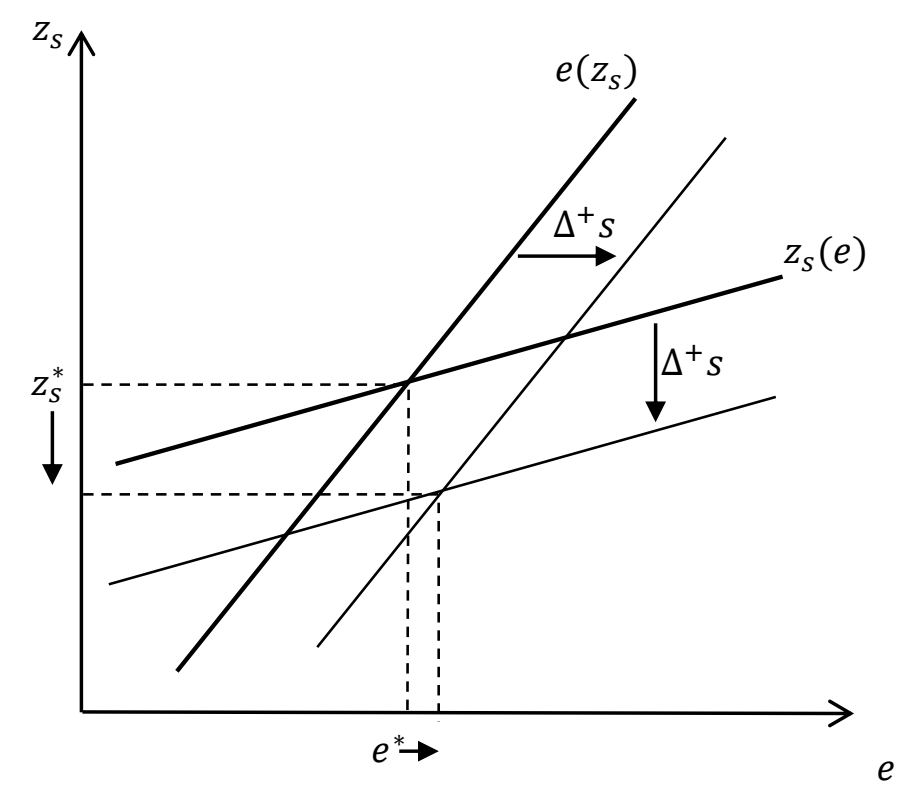

Fiqure 4

L'augmentation de $s$ conduit nécessairement à une augmentation de $e$ et à une réduction de $z_{s}$. Ces effets directs peuvent toutefois être renversés par le fait que les efforts entrepris aux deux périodes 
évoluent dans le même sens (effets indirects). La figure 4 représente une situation où l'incitation financière n'atteint pas son objectif dans la mesure où elle stimule des comportements bénéfiques à la santé pendant la durée du programme mais sans prolongements à long terme.

Il est intéressant de noter que notre résultat diffère de celui mis en évidence par Lee (2005) qui montre que lorsque la richesse et la santé sont complémentaires $\left(u_{12}>0\right)$ ), l'augmentation de la richesse de l'individu l'amène à renforcer sa propension à investir afin de réduire sa probabilité de développer une maladie. La différence entre notre conclusion et celle de Lee (2005) résulte de la structure des deux modèles: le coût de l'activité de prévention est contemporain à la réalisation éventuelle de la maladie et est exprimé en unités monétaires dans Lee (2005), alors que l'effort de comportement a des conséquences non-monétaires (mais en terme de qualité de vie) à la période qui précède la réalisation éventuelle de la perte dans notre modèle. Par ailleurs, Lee (2005) considère une augmentation exogène du revenu qui se réalise dans tous les états du monde (c'est-à-dire, que l'individu soit malade ou pas) alors que l'incitation financière que nous analysons n'est octroyée que dans l'état du monde favorable (celui où l'individu a atteint son objectif).

\section{Conclusion}

Les incitations financières octroyées lorsque les individus renoncent à certains comportements néfastes à la santé (tabagisme, sédentarité,...) permettent de contrebalancer les difficultés liées à ces changements d'habitudes par des bénéfices tangibles et immédiats. L'objectif étant que les individus prolongent leurs efforts - rendus moins pénibles car déjà amorcés - après la fin des programmes d'incitations. Dans ce travail, nous mettons en évidence la façon dont l'efficacité à long terme de ces programmes peut dépendre de l'interaction entre la richesse et la qualité de vie dans les préférences individuelles.

Dans le cadre de fonction d'utilité qui dépendent de la richesse des individus et de leur état de santé, la littérature empirique a montré que ces deux attributs étaient plutôt complémentaires ${ }^{13}$ (voir par exemple Viscusi et Evans (1990), Sloan et al. (1998), Carthy et al. (1999) et plus récemment Finkelstein, Luttmer et Notowidigdo (2013) $)^{14}$. Cette interaction entre la richesse et l'état de santé dans la fonction d'utilité individuelle correspond à la situation où l'utilité marginale de l'état de santé croît avec la richesse $\left(u_{12}>0\right)$. Dans notre article, le deuxième argument de la fonction d'utilité n'est pas l'état de santé mais une variable plus large - la qualité de vie - qui comprend l'état de santé. Si le même type d'interaction prévaut entre la richesse et la qualité de vie qu'entre la richesse et l'état de santé, notre travail montre que les programmes d'incitations financières afin de réduire les comportements néfastes à la santé peuvent avoir des effets opposés à ceux espérés, non pas si l'on considère les efforts fournis pour adopter un comportement sain pendant la durée du programme, mais si des prolongement à plus long terme de ces comportements sont visés.

\footnotetext{
${ }^{13}$ Notons toutefois que le résultat opposé a aussi parfois été mis en évidence (Evans et Viscusi (1991) et Edwards (2008)).

${ }^{14}$ Finkelstein, Luttmer et Notowidigdo (2009) proposent une synthèse des méthodologies adoptées dans ces différents travaux.
} 


\section{Bibliographie}

Briys E., Schlesinger H. 1990. "Risk aversion and the propensities for self-insurance and selfprotection.", Southern Economic Journal, 57: 458-467.

Carthy T., Chilton S., Covey J., Hopkins L., Jones-Lee M., Loomes G., Pidgeon N., Spencer A. 1999. “On the contingent valuation of safety and the safety of contingent valuation: Part 2-The CV/SG 'chained' approach.", Journal of Risk and Uncertainty, 17: 187-213.

Courbage C., Rey B. 2015. "Decision Thresholds and Changes in Risk for Preventive Treatment." Health Economics 25: 111-124.

Crainich D., Eeckhoudt L. et M. Menegatti. 2016. "Changing Risks and Optimal Effort.", Journal of Economic Behavior and Organization, 125: 97-106.

Edwards R., "Health Risk and Portfolio Choice." 2008. Journal of Business and Economic Statistics, 26(4): 427-485.008

Ehrlich I., Becker G. 1972. "Market Insurance, Self-Insurance and Self-Protection." Journal of Political Economy 80: 623-648.

Eeckhoudt L., Gollier C. 2005. "The impact of prudence on optimal prevention", Economic Theory, 26(4): 989-994.

Eeckhoudt L., Rey B., Schlesinger H. 2007. "A Good Sign for Multivariate Risk Taking.", Management Science 53: 117-124.

Evans W., Viscusi W. 1991. "Estimation of state-dependent utility functions using survey data", Review of Economics and Statistics, 73: 94-104.

Finkelstein E.A., Brown D.S., Brown D.R., Buchner D.M. 2008. "A Randomized Study of Financial Incentives to Increase Physical Activity among Sedentary Older Adults." Preventive Medicine 47(2): 182-87.

Finkelstein A., Luttmer E., Notowidigdo M. 1999. "Approaches to estimating the health state dependence of the utility function.", American Economic Review, 99: 116-121.

Finkelstein A., Luttmer E., Notowidigdo M. 2013. "What good is wealth without health: the effect of health on the marginal utility of consumption.", Journal of the European Economic Association, 11 (S1): 221-258.

Halpern S.D., French B., Small D.S. et al. 2015. "Randomized Trial of Four Financial-Incentive Programs for Smoking Cessation." New England Journalof Medicine 372:2108-2117.

Jindapon P., Neilson W.2007. "Higher-Order Generalizations of Arrow-Pratt and Ross Risk Aversion: a Comparative Statics Approach", Journal of Economic Theory, 136(1): 719-728. 
John L.K., Loewenstein G., Troxel A.B., Norton L., Fassbender J.E., Volpp K.G., et al. 2011. "Financial Incentives for Extended Weight Loss: A Randomized, Controlled Trial." Journal of General Internal Medicine 26: 621-26.

Jullien B., Salanié B., Salanié F. 1999. "Should more risk-averse agents exert more effort?" Geneva Papers on Risk and Insurance Theory, 24: 19-28.

Lee, K. 2005. "Wealth Effects on Self-Insurance and Self-Protection against Monetary and Nonmonetary Losses." The Geneva Risk and Insurance Review, 30: 147-159.

Menegatti, M. 2014. "Optimal Choice on Prevention and Cure: a New Economic Analysis." European Journal of Health Economics 15(4): 363-372.

Organisation Mondiale de la Santé. 2015. Rapport sur la santé en Europe 2015. Les cibles et au-delàatteindre les nouvelles frontières des bases factuelles. Copenhague, Bureau regional de l'OMS pour l'Europe.

Paul-Ebhohimhen V. et A. Avenell. 2008. "Systematic review of the use of financial incentives in treatments for obesity and overweight." Obesity Review, 9(4): 355-357.

Sloan F., Viscusi W., Chesson W., Conover C., Whetten-Goldstein K. 1998. "Alternative Approaches to Valuing Intangible Health Losses: the Evidence for Multiple Sclerosis.", Journal of Health Economics, 17: 475-97.

Spahn J.M., Reeves R.S., Keim K.S., Laquatra I., Kellogg M., Jortberg B., Clark N.A. 2010. "State of the Evidence Regarding Behavior Change Theories and Theories in Nutrition Counseling to Facilitate Health and Food Behavior Change." Journal of the American Dietetic Association 110: 879-91.

Tappin D., Bauld L., Purves D., Boyd K., Sinclair L., MacAskill S. et al. 2015. "Financial incentives for smoking cessation in pregnancy: randomised controlled trial." BMJ350:h134.

Viscusi W., Evans W. 1990. "Utility functions that depend on health status: estimates and economic implications.", American Economic Review, 80: 353-374.

Volpp K.G., John L.K., Troxel A.B., Norton L., Fassbinder J., Loewenstein G. 2008. "Financial IncentiveBased Approaches for Weight Loss." Journal of the American Medical Association 300: 2631-37.

\section{Annexe 1}

Dans l'article, nous avons fait l'hypothèse que les efforts effectués par les individus ne modifiaient pas leur niveau de richesse. Cela peut être le cas si l'on considère la pratique de sports. Dans cette annexe, nous généralisons le modèle en supposant que l'effort effectué par l'individu à chaque période affecte non seulement sa qualité de vie mais aussi sa richesse.

Les effets financiers résultant de l'adoption de comportements sains varient d'un cas à l'autre. Par exemple, l'arrêt du tabagisme peut nécessiter l'achat de substituts et améliorer son alimentation peut impliquer l'achat d'aliments plus coûteux. Mais ces deux mêmes actions peuvent aussi générer des gains financiers: les individus effectuent des économies en n'achetant plus de tabac et en 
n'achetant plus certains aliments néfastes à la santé. II est donc difficile de déterminer a priori la façon dont la richesse devrait varier avec les efforts effectués.

Nous développons ici le cas où les efforts de comportement permettent aux individus d'économiser de l'argent. Cette hypothèse ne modifie pas le résultat principal de l'article. L'hypothèse inverse conduit à des résultats plus mitigés et n'est pas discutée dans le cadre de cette annexe.

Nous supposons que La fonction $g($.$) indique la conséquence financière de l'effort de$ comportement. Compte tenu de la discussion ci-dessus, nous supposons que $g^{\prime}()>$.0 . L'individu choisit désormais les niveaux d'efforts $e, z_{S}$ et $z_{s}$ qui maximisent l'expression ci-dessous :

$$
\begin{aligned}
A=u(w+g(e) & , h-e) \\
& +(1-p(e))\left[u\left(w+s+g\left(z_{s}\right), h-\alpha\left(e, z_{s}\right)\right)+\left(1-q\left(z_{s}\right)\right) E U(\widetilde{L})+q\left(z_{s}\right) E U(\widetilde{H})\right] \\
& +p(e)\left[u\left(w+g\left(z_{s}\right), h-\varphi\left(z_{s}\right)\right)+\left(1-q\left(z_{s}\right)\right) E U(\widetilde{L})+q\left(z_{s}\right) E U(\widetilde{H})\right]
\end{aligned}
$$

$S$ et $\mathcal{S}$ s'écrivent désormais : $S=\left(w+s+g\left(z_{S}\right), h-\alpha\left(e, z_{S}\right)\right)$ et $S=\left(w+g\left(z_{s}\right), h-\varphi\left(z_{s}\right)\right)$

Les conditions de premier ordre qui définissent les niveaux optimaux des efforts $e^{*}, \mathrm{z}_{s}^{*}$ et $z_{s}^{*}$ s'écrivent alors:

$$
\begin{gathered}
A_{e}=g^{\prime}\left(e^{*}\right) u_{1}\left(w+g(e), h-e^{*}\right)-u_{2}\left(w+g(e), h-e^{*}\right)-p^{\prime}\left(e^{*}\right)[E U(S)-E U(S)] \\
\quad-\left(1-p\left(e^{*}\right)\right) \alpha_{e}\left(e^{*}, z_{s}\right) u_{2}(S)=0 \\
A_{z_{S}}=g^{\prime}\left(\mathrm{z}_{s}^{*}\right) u_{1}(S)-\alpha_{z}\left(e, \mathrm{z}_{S}^{*}\right) u_{2}(S)-q^{\prime}\left(\mathrm{z}_{s}^{*}\right)[E U(\widetilde{L})-E U(\widetilde{H})]=0 \\
A_{z_{s}}=g^{\prime}\left(z_{s}^{*}\right) u_{1}(S)-\varphi^{\prime}\left(z_{s}^{*}\right) u_{2}(S)-q^{\prime}\left(z_{s}^{*}\right)[E U(\widetilde{L})-E U(\widetilde{H})]=0
\end{gathered}
$$

La même analyse que celle proposée dans l'article peut alors être développée.

L'effet direct de l'incitation financière sur l'effort de comportement à la période 0 (c'est-à-dire le déplacement de la fonction de réaction $e\left(z_{S}\right)$ avec $s$ ) est donné par l'expression :

$$
\frac{\delta A}{\delta e \delta s}=A_{e s}=-p^{\prime}\left(e^{*}\right) u_{1}(S)-\left(1-p\left(e^{*}\right)\right) \alpha_{e}\left(e^{*}, z_{S}\right) u_{12}(S)
$$

qui permet de montrer que :

$$
u_{12} \geq 0 \Rightarrow A_{e s}>0
$$

L'effet direct de l'incitation financière sur l'effort de comportement à la période 1 lorsque l'objectif du programme a été atteint à la période précédente ( $c$ 'est-à-dire le déplacement de la fonction de réaction $z_{s}(e)$ avec $\left.s\right)$ est donné par :

$$
\frac{\delta A}{\delta z_{S} \delta S}=A_{z_{S} s}=g^{\prime}\left(\mathrm{z}_{S}^{*}\right) u_{11}(S)-\alpha_{z}\left(e, \mathrm{z}_{S}^{*}\right) u_{12}(S)
$$

Dans ce cas, $u_{12}>0$ n'est plus une condition nécessaire et suffisante (c'est le cas si l'effort n'a pas d'effets financiers; voir page 9) mais seulement une condition suffisante pour que la fonction de réaction $z_{S}(e)$ se déplace vers le bas lorsque $s$ augmente :

$$
u_{12} \geq 0 \Rightarrow A_{z_{S} s}<0
$$


L'existence des conséquences financières de l'effort augmente donc les cas de figure où l'augmentation de l'incitation financière $s$ conduit à un effet direct négatif sur l'effort effectué à la période 1 lorsque l’objectif du programme a été atteint à la période 0 .

Enfin, nous déterminons le signe des fonctions de réaction compte tenu de la nouvelle hypothèse effectuée :

$$
\begin{aligned}
& A_{e z_{s}}=-p^{\prime}(e)\left[g^{\prime}\left(\mathrm{z}_{s}^{*}\right) u_{1}(S)-\alpha_{z}\left(e, \mathrm{z}_{s}^{*}\right) u_{2}(S)-q^{\prime}\left(\mathrm{z}_{s}^{*}\right)[E U(\tilde{L})-E U(\widetilde{H})]\right] \\
& \quad-(1-p(e))\left[\alpha_{e z}\left(e, \mathrm{z}_{s}^{*}\right) u_{2}(S)+\alpha_{e}\left(e, \mathrm{z}_{s}^{*}\right) g^{\prime}\left(\mathrm{z}_{s}^{*}\right) u_{12}(S)-\alpha_{z}\left(e, \mathrm{z}_{s}^{*}\right) \alpha_{e}\left(e, \mathrm{z}_{s}^{*}\right) u_{22}(S)\right]
\end{aligned}
$$

Puisque $A_{z_{S}}=0$ à l'équilibre, la première ligne de l'expression s'annule et le signe de $A_{e z_{S}}$ correspond à celui de la seconde ligne qui est positif compte tenu des hypothèses effectuées. Nous pouvons donc en conclure que $A_{e z_{s}}>0$, ce qui implique que les fonctions de réaction $e\left(z_{s}\right)$ et $z_{s}(e)$ sont croissantes.

Comme lorsque l'effort de comportement n'a pas d'effet financier (voir le modèle développé dans l'article), $u_{12} \geq 0$ peut donc aussi amener à des situations où l'incitation financière à l'adoption de comportements sains a des effets contre-productifs à long terme. Il est à noter que l'hypothèse de conséquences financières positives des efforts de comportement renforce cet effet dans la mesure où $u_{12} \geq 0$ n'est plus une condition nécessaire et suffisante mais simplement une condition suffisante pour que l'augmentation de $s$ ait un impact direct négatif sur la fonction de réaction $z_{s}(e)$. 